\section{Usefulness of lower gastrointestinal endoscopy on suspicion of an iatrogenic sigmoid perforation caused by an aorto-bifemoral prosthetic bypass}

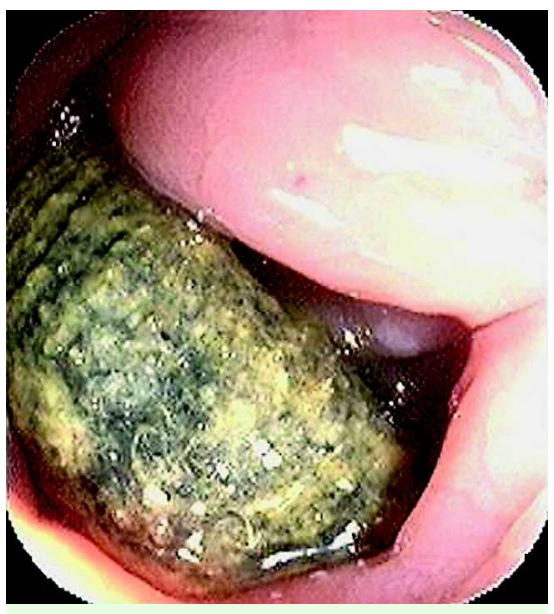

Fig. 1 Aorto-bifemoral prosthesis inside the sigmoid colon.

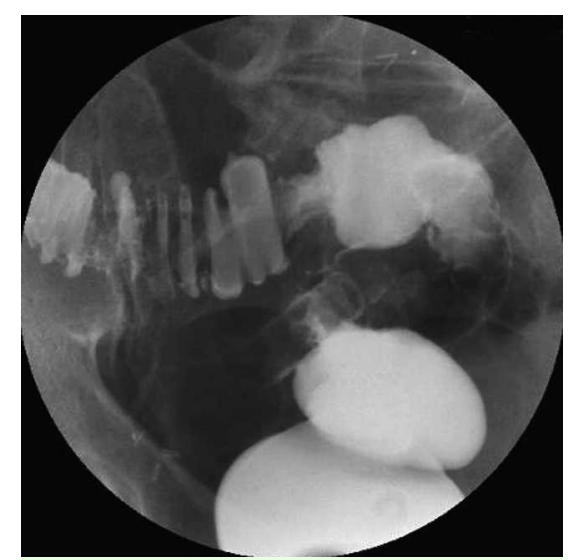

Fig. 2 Double-contrast colon radiograph showing the middle part of the stent inside the sigmoid colon.

Prosthetic graft infection occurs in up to $6.0 \%$ of patients who undergo aortic surgery and is associated with a high mortality rate $(20-75 \%)$ [1]. Vascular-enteric fistulas (VEF) most commonly involve the third or fourth portion of the duodenum ( $87 \%$ of cases), rarely the colon $(2 \%)$ $[2,3]$.
A 76-year-old man was admitted to our institution because of sepsis, most likely induced by graft infection. The patient had received an aorto-bifemoral bypass for treatment of an abdominal aortic aneurysm 10 months earlier. The postoperative course was characterized by sepsis (Pseudomonas aeruginosa). Before admission the patient reported episodes of rectorrhagia; angio-CT showed that the left graft limb had perforated straight through the sigmoid colon. Colonoscopy allowed the vascular stent to be located in the sigmoid colon ( $\bullet$ Fig. 1,2). The proximal and distal extremities of the stent were not recognizable, since the stent had migrated into the extraluminal compartment. Exploratory surgery was conducted and revealed that the vascular stent had become displaced through the distal sigmoid colon, leading to perforation of the wall. The affected part of the sigmoid was resected together with the graft limb, with creation of a temporary Hartmann's diversion. We suggest performing lower gastrointestinal endoscopy early on suspicion of VEF in patients who have had a vascular stent placed and who present recurrent infectious episodes and low gastrointestinal bleeding of unexplained origin.

Endoscopy_UCTN_Code_CCL_1AD_2AH

Endoscopy_UCTN_Code_CCL_1AD_2AJ

\section{B. Mangiavillano, G. Mezzi, P. A. Testoni} Division of Gastroenterology and Gastrointestinal Endoscopy Unit, Vita-Salute San Raffaele University, San Raffaele Scientific Institute, Milan, Italy

\section{References}

1 Ricco JB InterGard Silver Study Group. InterGard silver bifurcated graft: features and results of a multicenter clinical study. J Vasc Surg 2006; 44: 339-346

2 Bunt TJ. Synthetic vascular graft infections. II. Graft-enteric erosions and graft-enteric fistulas. Surgery 1983; 94: 1-9

3 Tsunekawa T, Ogino H, Minatoya $K$ et al. Masked prosthetic graft to sigmoid colon fistula diagnosed by 18 -fluorodeoxyglucose positron emission tomography. Eur J Vasc Endovasc Surg 2007; 33: 187-189

Bibliography

DOI 10.1055/s-2007-995799

Endoscopy 2008; 40: E170

(c) Georg Thieme Verlag KG Stuttgart · New York . ISSN 0013-726X

Corresponding author

\section{B. Mangiavillano, MD}

Division of Gastroenterology and Gastrointestinal Endoscopy

University Vita-Salute San Raffaele

Scientific Institute San Raffaele

Via Olgettina 62

20132 Milan

Italy

Fax: +39-02-26432504

mangiavillano.benedetto@hsr.it 